\title{
Are Triggering Rates of Labquakes Universal? Inferring Triggering Rates From Incomplete Information.
}

\author{
Jordi Baró, Jörn Davidsen \\ Complexity Science Group, Department of Physics and Astronomy, University of Calgary, Canada
}

September 6, 2018

\begin{abstract}
The acoustic emission activity associated with recent rock fracture experiments under different conditions has indicated that some features of event-event triggering are independent of the details of the experiment and the materials used and are often even indistinguishable from tectonic earthquakes. While the event-event triggering rates or aftershock rates behave pretty much identical for all rock fracture experiments at short times, this is not the case for later times. Here, we discuss how these differences can be a consequence of the aftershock identification method used and show that the true aftershock rates might have two distinct regimes. Specifically, tests on a modified Epidemic Type Aftershock Sequence model show that the model rates cannot be correctly inferred at late times based on temporal information only if the activity rates or the branching ratio are high. We also discuss both the effect of the two distinct regimes in the aftershock rates and the effect of the background rate on the inter-event time distribution. Our findings should be applicable for inferring event-event triggering rates for many other types of triggering and branching processes as well.
\end{abstract}

\section{Event-event triggering \& aftershocks}

Many striking features of physical, geophysical, biological or social processes can be portrayed as patterns or clusters of localized events. Specific examples include magnetization processes [1, 2, 3, martensitic transformations 4], fracture processes [5, 6, 7, 8, 9, 10, natural or induced earthquakes [11, 12, 13, 14, solar flares [15, 16, extreme bursts in the solar wind [17, 18, the spread of infections [19, extinctions of species [20, 21, 22, neural spikes 223, 24, booms and bursts of markets and economies [20, 25, 26, 27, 28, 29, 30, media coverage 31] - to name a few. A generic attribute in all these cases is that one event can trigger or somehow induce another one to occur - or possibly numerous further events. One of the most prominent examples of such event-event triggering are aftershocks [32, 33, 34, 35, 36, 37, 13. Aftershock sequences are characterized by timevarying (local) event rates, which are often empirically found to approximately follow across a wide range of scales and systems from friction and fracture to socio-economic systems [35, 38, 39, 40, 41, 7, 9, 10, 16, 27, 28, 29, 30, 31] — the Omori-Utsu (OU) relation,

$$
r(t)=\frac{K}{(t+c)^{p}} \equiv \frac{1}{\tau(t / c+1)^{p}},
$$

first proposed for earthquakes [42. Here, $t$ measures the time after the triggering event, $p$ is typically close to 1 ( $p \gtrsim 1$ if one only considers directly triggered events [40]) and $\tau \equiv c^{p} / K . K$ is typically found to increase with the energy of the trigger though the exact 
dependence of $K, c$ and hence $\tau$ on different parameters is an active field of research [13].

The OU relation with $p \approx 0.7$ has in particular been observed in acoustic emission (AE) experiments of rock fracture across a range of different materials and conditions [7, 43, 44, denoting some sort of universality in the response of disordered materials under mechanical stress, an hypothesis already suggested by the scale invariance in other physical and statistical relations 45, 46, 47]. Yet, a $p$-value significantly lower than 1 implies that the number of events directly or indirectly triggered by a single event is infinite. Indeed, a more recent study of rock fracture experiments using a more reliable technique to identify triggered events has shown that there are significant deviations from the OU relation at late times with a steeper decay that ensures that the number of triggered events is finite [48. Identifying the aftershocks is a general challenge for all triggering processes since a detailed or "fundamental" knowledge of the underlying microscopic dynamics and causal information is typically not available [49]. In the case of earthquakes, the most reliable methods to identify triggering relations use spatio-temporal correlations between events [35, 39, 50, 51]. In the absence of spatial information - as it is the case for previous rock fracture experiments $7,43,44$, this is not an option and one has to rely on the measurement of the whole activity rate after each event $\mathbf{7 , 5 2}$. This technique can lead to a strong bias in the estimation of triggering rates in cases where either the number of triggered events or the background rate of events activated by other mechanisms is high, or both, as we show explicitly here.

Specifically, in this paper we aim to quantify the bias of this technique and establish under which conditions it can serve as a reasonable estimator for the triggering rates. We test it against synthetic catalogs generated by a modified Epidemic-Type Aftershock Sequence (ETAS) model with a triggering rate characterized by two power laws, as observed in the most recent rock fracture experiments [48. We address the possibility that the low experimental OU exponent ( $p$ values) and some other inconsistent results observed in the previous rock fracture experiments [7, 43, 44, are a consequence of the hidden complexity of the triggering process. First, we formulate triggering in terms of branching processes. The true direct and compound triggering rates can be estimated reliably only under certain conditions. Instead, one is often limited to less reliable estimators such as those based on the mean aftershock sequence rates (MASR) and the distribution of waiting times (DWT) to extract the properties of the triggering process. We discuss the limitations of such methods. Next, we introduce the modified ETAS model with the triggering rates characterized by two power laws as observed in rock fracture experiments with spatio-temporal information [48. Considering that only magnitude and temporal information is available, we interpret the measured MASR in terms of direct and compound triggering rates, and identify the parameters giving rise to different power-law regimes in the DWT. Finally, we discuss whether these numerical results can provide an explanation for anomalous features in the experimental results.

\section{Event-event triggering represented as a branching process}

The study of systems exhibiting localized events and triggering between them can be cast in the language of point processes [53, [54, 55, 56, 13]. A stochastic point process is fully determined by a function called the intensity, which quantifies the probability of occurrence of an event of size $M$ at time $t$ and at location $\vec{z}$ :

$$
\mu(t, \vec{z}, M):=\operatorname{Prob}\{\text { event of size } M \text { at } t, \vec{z}\} d t d \vec{r} d M .
$$

The measured activity rate can vary over time due to an explicit temporal variation of 
external parameters, and/or as a consequence of previous activity. In the latter case, the intensity depends explicitly on the history of the point process $\left(\mathcal{H}_{t}:=\right.$ all events $i ; t_{i}<$ $t\})$. The exact intensity $\left(\mu\left(t, \mathcal{H}_{t}\right)\right)$ of a process exhibiting triggering involves in general a complex contribution of the whole history $\mathcal{H}_{t}$. In a simplified approach, the contribution to the intensity of each past event can be linearized in a Hawkes self-exciting point process [57]:

$$
\mu\left(t, \mathbf{z}, m \mid \mathcal{H}_{t}\right)=\mu_{0}(t, m)+\sum_{i \in \mathcal{H}_{t}} \phi\left(t-t_{i}, \vec{z}-\vec{z}_{i}, m \mid M_{i}\right)
$$

Stochastic Hawkes processes can be reinterpreted as the outcome of branching processes where each event is either a background event or has a single parent. Given a background event $(G=0)$, a sequence of first generation events $(G=I)$ can be triggered, after a time difference $\tau$ and at relative position $\vec{r}$ from the background event, according to an intensity factor represented by the triggering kernel $\phi\left(\tau, \vec{r}, m \mid M_{i}\right)$. Each event in the first generation can itself trigger a sequence of second generation events $(\mathrm{G}=\mathrm{II})$ with the same relative kernel $\phi\left(\tau, \vec{r}, m \mid M_{i}\right)$, and so on until a whole triggering cascade or tree is generated up to some $n$-th generation that does not trigger further events. The major physical constraint to the model is the stability of the branching process, requiring that the average number of events directly triggered from a single parent - computed as the average branching ratio: $n_{b}=\left\langle\int d \tau \int d \vec{r} \int d m \int d m^{\prime} \phi\left(\tau, \vec{r}, m^{\prime} \mid m\right)\right\rangle$ - has to be lower than one. Triggering trees can be spatially and temporally overlapping, generating a complex triggering forest difficult to disentangle in practice.

\subsection{Direct and compound event-event triggering rates}

The linear Hawkes model is useful for both the development of forecasting tools 58, 59, 60. 61 and the deep understanding of the fundamental physics behind avalanche processes [62, 63, 64, 65]. Both purposes require a reliable estimation of the triggering kernel. If the actual pairwise parent-child relations are retrievable, or can be estimated from declustering techniques [66, 50, 35, 51, 67, , one can measure the direct (or bare 68]) triggering rates and use them as a good estimator for the triggering kernel: $\widehat{\phi}\left(\tau, \vec{r}, m^{\prime} \mid m\right)$.

In some cases one has to deal with time series without spatial information, or situations where the spatial kernel is too spread to retain meaningful information. One is left with the marginal temporal point process with a triggering kernel $\phi\left(\tau, m \mid m_{0}\right)$. The compound (or dressed [69]) triggering rates are the expected temporal activity during the span of a triggering cascade generated from a background event (the root of the tree) of magnitude $m_{0}$ at $t_{0}$. The compound triggering rates can be computed from the direct triggering rates as:

$$
\Phi_{c}\left(\tau, m \mid m_{0}\right):=\sum_{G=I, I I, \ldots}^{\infty} \Phi_{G}\left(\tau, m \mid m_{0}\right)
$$

where $\tau=t-t_{0}$ and $\Phi_{G=I}$ corresponds to the direct rates from the background event, $\Phi_{G=I I}$ designates the rate of events triggered by all first generation events, etc.. Under certain conditions one can calculate the compound rates analytically, or at least find an approximate solution, as for the case exposed below. The magnitude of triggered events is usually assumed as an independent variable: $\phi\left(\tau, m \mid m_{0}\right)=\rho(m) \phi\left(\tau \mid m_{0}\right)$, where $\rho(m)$ is the distribution of magnitudes. In such cases, the intensity of the first generation $(G=I)$, equivalent to the kernel from the triggering background event, can be written as:

$$
\Phi_{I}\left(\tau, m \mid m_{0}\right) \equiv \rho(m) \phi\left(\tau \mid m_{0}\right)
$$

and each one of the higher order generations contribute to the intensity as:

$$
\Phi_{G}\left(\tau, m \mid m_{0}\right)=\rho(m) \int_{m_{c}}^{\infty} d m^{\prime} \int_{0}^{\tau} d t^{\prime} \Phi_{G-1}\left(t^{\prime} \mid m_{0}\right) \phi\left(\tau-t^{\prime} \mid m^{\prime}\right) \rho\left(m^{\prime}\right)
$$


where $t^{\prime}$ and $m^{\prime}$ are the occurrence time and magnitude of the events from the $(G-$ 1 )'th generation originated by the mainshock $\left(m_{0}, t_{0}\right)$, and $m^{\prime}$ is distributed according to $\rho\left(m^{\prime}\right)$. If the kernel can be further separated between the temporal and productivity term: $\phi\left(\tau \mid m^{\prime}\right):=k\left(m^{\prime}\right) \widetilde{\phi}(\tau)$, the branching ratio is simplified as: $n_{b}=\int k\left(m^{\prime}\right) \rho\left(m^{\prime}\right) d m^{\prime}$, and each element is directly triggered by the previous generation:

$$
\Phi_{G}\left(\tau \mid m_{0}\right)=n_{b} \int_{0}^{\tau} d t^{\prime} \Phi_{G-1}\left(t^{\prime}, m_{0}\right) \widetilde{\phi}\left(\tau-t^{\prime}\right)
$$

Substituting Eq. (5) in the generic recurrence the triggering rates of generation $G$ can be expressed as a series of convolution operations $(*)$ as:

$$
\Phi_{G}\left(\tau \mid m_{0}\right)=K\left(m_{0}\right) n_{b}{ }^{g}\left(\widetilde{\phi}_{1} * \widetilde{\phi}_{2} * \ldots * \widetilde{\phi}_{g}\right)
$$

This recurrent expression can be solved in the Laplace transformed space. Given $\widetilde{\psi}(s):=$ $\mathcal{L}(\widetilde{\phi}(y))$ the compound rates can be found as:

$$
\Phi_{c}\left(\tau \mid m_{0}\right)=K\left(m_{0}\right) \sum_{g=1}^{\infty} \mathcal{L}^{-1}\left(\left(n_{b} \widetilde{\psi}(s)\right)^{g}\right)
$$

\subsection{Mean aftershock sequence rates}

In the absence of any information regarding the precise topological structure of the branching process we can still try to estimate the triggering kernel under certain conditions. The branching nature of the model, and the independence between terms, impose Markovian correlations in the parent-child relationship. All triggering branches are independent and, thus, triggering trees can be considered independently of the generation of the parent without loss of generality. Under these premises, we can consider all triggering trees originated from any event as statistically equivalent. Thus, we can measure the activity rates conditioned to the presence of a trigger or mainshock $\left(t_{0}, m_{0}\right)$ - the mean aftershock sequences rates (MASR) - as the expected density of events:

$$
\operatorname{MASR}\left(\tau, m \mid m_{0}\right):=\left\langle\rho\left(t-t_{i}=\tau, m, M_{i}=m_{0}\right)\right\rangle
$$

In general, the measurement of MASR corresponds to the compound rates plus the contribution of all events without a causal connection to the mainshock. If the branching ratio is low, we can consider this second contribution as the independent activity:

$$
\operatorname{MASR}\left(\tau \mid m_{0}\right) \approx \Phi_{c}\left(\tau \mid m_{0}\right)+\left\langle\mu_{0}(t)+\int d t^{\prime} \mu\left(t^{\prime}\right) \phi\left(t-t^{\prime} \mid m_{0}\right)\right\rangle \approx \Phi_{c}\left(\tau \mid m_{0}\right)+\langle\mu(t)\rangle
$$

Under certain conditions the MASR serve as a good approximation to the compound or even the direct rates. If the background activity is low enough to isolate independent triggering trees, the contribution of independent events is small and $\operatorname{MASR}\left(\tau \mid m_{0}\right) \approx \Phi_{c}\left(\tau \mid m_{0}\right)$. Furthermore, if the branching ratio is low enough to neglect secondary triggering as a major contribution to the triggering rates, compound and direct rates are similar and hence $\Phi_{c}\left(\tau \mid m_{0}\right) \approx \phi\left(\tau \mid m_{0}\right)$.

\subsection{The Distribution of Waiting Times}

Finally, we shall mention the more naive approach to study triggering based on the distribution of waiting times (DWT) or times between consecutive events. Since the DWT is a memoryless measurement, different point process (with and without correlations) can give rise to similar distributions. It is advised to use the DWT with caution when assessing the 
presence of triggering, and to use more reliable techniques instead [70, 71, 72, 73, 74].

Specifically, different phenomena can give rise to power-law regimes in the DWT. For a Poisson process, all events are independent and their DWT renders a decaying exponential with a characteristic rate (e.g. $\mu_{0}$ in Eq. 3). Yet, if this rate varies over time, the compound DWT corresponds to a superposition of exponential distributions. If the rate starts from zero $\left(\mu_{0}(t=0)=0\right)$ and increases with time, we can always expand the temporal dependence of the background rate in a power series around the origin: $\lim _{t \rightarrow 0+} \mu_{0}(t) \approx t^{1 / \xi}$. It can be shown that, for long waiting times, the DWT for this process of independent events will be: $\operatorname{DWT}(\delta) d \delta \propto \delta^{-\xi-2} d \delta[7,75,76,77]$.

On the other hand, a power-law DWT can also emerge from triggering. Typically, the presence of triggering is identified as an anomalous behavior in the distribution of short waiting times. Direct triggering rates decaying in time as a power-law $r\left(t-t_{i}\right) \propto\left(t-t_{i}\right)^{-p}$ - such as the Omori-Utsu relation in Eq. (1) - return also a power-law with an exponent $2-1 / p$ [6, 77, 38. However, some experimental measurements also display discrepancies with this exact relation [7, 4, 78. Finally, when power-law triggering processes coexist with a time-dependent background rate we can find a double power-law DWT fulfilling certain scaling relations [76, 7].

\section{Triggering Models from Empirical Data}

One of the most studied triggering processes are aftershock sequences in seismology. Seismic activity increases after major earthquakes, a phenomenon consistent with the idea that this event - often called a main shock - triggered other ones. In $1894 \mathrm{~F}$. Omori realized that the rate of the triggered earthquakes - the aftershocks - after the 1891 Nobi main shock decayed in time following a power-law relation with exponent $p \approx 1$ [4]. The modified Omori-Utsu relation [38, as stated in Eq. (1), presents a good approximation for the activity rates after almost all major earthquakes in recent history. The size of an earthquake is quantified by the magnitude $m$, a logarithmic measure of the seismic moment released by the slip associated with an earthquake. In 1944 Gutenberg and Richter 79] established that the number of earthquakes above a certain magnitude $m$ approximately behaves as $N_{>}(m) \sim 10^{-b m}$, equivalent to a power-law distribution of the seismic moment or an exponential distribution of magnitudes:

$$
\rho(m) d m=b \ln (10) 10^{-b\left(m-m_{c}\right)} d m \quad \text { for } \quad m_{c} \leq m
$$

where $m_{c}$ corresponds to the magnitude of completeness of the given catalog. The value of $b$ is close to unity and Eq. (12) extends down to magnitudes as low as $m=-4.4$ [80]. Typically, magnitudes can be considered to be independent 81,82 . There exists, however, a well established relationship between the magnitude of main shocks and the number of their aftershocks $N_{\mathrm{AS}}$. The productivity relation of aftershocks states that $N_{\mathrm{AS}}$ scales with the magnitude of the mainshock $m_{0}$ as 38 :

$$
N_{\mathrm{AS}}\left(m_{0}\right) \propto 10^{\widetilde{\alpha}} m_{0}
$$

This relation implies that the parameter $K$ in the OU relation (1) is not a constant and instead scales with the magnitude of the mainshock as: $K\left(m_{0}\right)=k 10^{\alpha m_{0}}$ and, in this case, $\alpha \equiv \widetilde{\alpha}$. Yet, recent studies of earthquake catalogs indicate that the productivity relation and the Omori-Utsu relation might need to be augmented and $\alpha \neq \widetilde{\alpha}[13$. 


\subsection{The Epidemic-Type Aftershock Sequence (ETAS) model of earthquakes}

The three statistical relations of seismology stated in Eqs. (11213) can be used to define a branching process 1 , commonly known as the Epidemic Type Aftershock (ETAS) Model [83, where all the explicit dependences can be separated as:

$$
\phi\left(m, \tau, \mathbf{r} \mid m_{0}\right)=\rho(m) K\left(m_{0}\right) \widetilde{\phi}_{T}(\tau) \widetilde{\phi}_{R}(\mathbf{r}) \text { with }\left\{\begin{array}{l}
\rho(m)=b \ln (10) 10^{b\left(m_{c}-m\right)} \\
K\left(m_{0}\right)=k 10^{\alpha m_{0}} \\
\widetilde{\phi}_{T}(\tau)=\theta C^{\theta}(C+\tau)^{-1-\theta}
\end{array}\right.
$$

where $m_{0}$ is the magnitude of the mainshock and $\tau=t-t_{0}$. The average branching ratio is given by: $n_{b}=k \frac{b}{b-\alpha} 10^{\alpha m_{c}}$. In the branching process approach, the number of triggered events is sampled as a Poisson variable with rate $K\left(m_{0}\right)$.

\subsection{The modified ETAS model for rock fracture}

Many physical processes exhibit statistical features similar to those summarized in Eqs. (1) 12 13) for seismicity. Specifically, the ETAS model describes remarkably well some aspects of the temporal sequences of acoustic emission ( $\mathrm{AE}$ ) events recorded during the failure of rocks and porous materials under compression [7]. However, the full spatio-temporal triggering cascades, which have only become accessible very recently, in AE experiments of rock fracture reveal a more complex triggering kernel than the standard ETAS model [48. While the event magnitudes appear to be independent $\left(\phi\left(m, \tau \mid m_{0}\right)=\rho(m) \phi\left(\tau \mid m_{0}\right)\right)$, empirical evidence suggests that there is a characteristic time associated with the triggering rates that scales with the magnitude of the mainshock. Similar behavior has also been observed very recently in earthquake catalogs from Southern California [40, 13. Thus, the term $\phi\left(\tau \mid m_{0}\right)$ cannot be separated in independent terms $K\left(m_{0}\right) \phi(\tau)$. Specifically, the following scaling form has been observed: $\phi\left(\tau \mid m_{0}\right) \sim 10^{\alpha m_{0}} \widetilde{\phi}\left(10^{-\alpha_{\tau} m_{0}} \tau\right)$ with $\alpha_{\tau}=0.5$ [48]. Furthermore, the short time regime, which is constant in the standard ETAS model, is better fitted by a generic power law with an exponent $0 \leq p_{1}<1$. In summary, the scaling function exhibits a transition from this power-law regime $\widetilde{\phi}(x) \sim x^{-p_{1}}$ below a characteristic value $x_{c}$ of rescaled time, towards the standard $\widetilde{\phi}(x) \sim x^{-p_{2}}$ with $p_{2}>1$. In order to implement a modified ETAS model able to reproduce the empirical observations, we need to impose a branching ratio $n_{b} \leq 1$ for stability reasons, and set $p_{1}<1$ and $p_{2}>1$ to be integrable over the whole temporal domain. We define the positive parameters $\theta_{1}:=1-p_{1}$ and $\theta_{2}:=p_{2}-1$ for convenience. By normalizing the kernel and imposing the continuity constrain at $x_{ \pm}=x_{c}$ the scaling relation has the explicit form:

$$
\frac{1}{x_{c}} \widetilde{\phi}\left(x / x_{c}\right) d x=\frac{d x}{x_{c}}\left(\frac{\theta_{1} \theta_{2}}{\theta_{2}+\theta_{1}}\right) \begin{cases}\left(\frac{x_{c}}{x}\right)^{1-\theta_{1}} & \text { for } \quad 0<x / x_{c} \leq 1 \\ \left(\frac{x_{c}}{x}\right)^{1+\theta_{2}} & \text { for } \quad x / x_{c} \geq 1\end{cases}
$$

Considering the same $K\left(m_{0}\right)$ used in Eq. (14) and the definition of the productivity law stated in Eq. (13), the total number of events triggered by a mainshock of magnitude $m_{0}$ now scales with the compound productivity exponent $\widetilde{\alpha}=\alpha+\alpha_{\tau}$. Given the temporal and mainshock-magnitude dependent kernel $\phi\left(\tau \mid m_{0}\right)$ the scaling function $\widetilde{\phi}\left(x / x_{c}\right)$ from Eq. (15) can be retrieved as:

$$
\widetilde{\phi}\left(\frac{10^{-\alpha_{\tau} m_{0}} \tau}{x_{c}}\right)=\frac{x_{c}}{k} 10^{-\alpha m_{0}} \phi\left(\tau \mid m_{0}\right) .
$$

\footnotetext{
${ }^{1}$ Notice that the exponent $1+\theta$ and the $p$ in the OU relation (1) measured from the compound rates may differ [52], as can be derived from Eq. (9).
} 


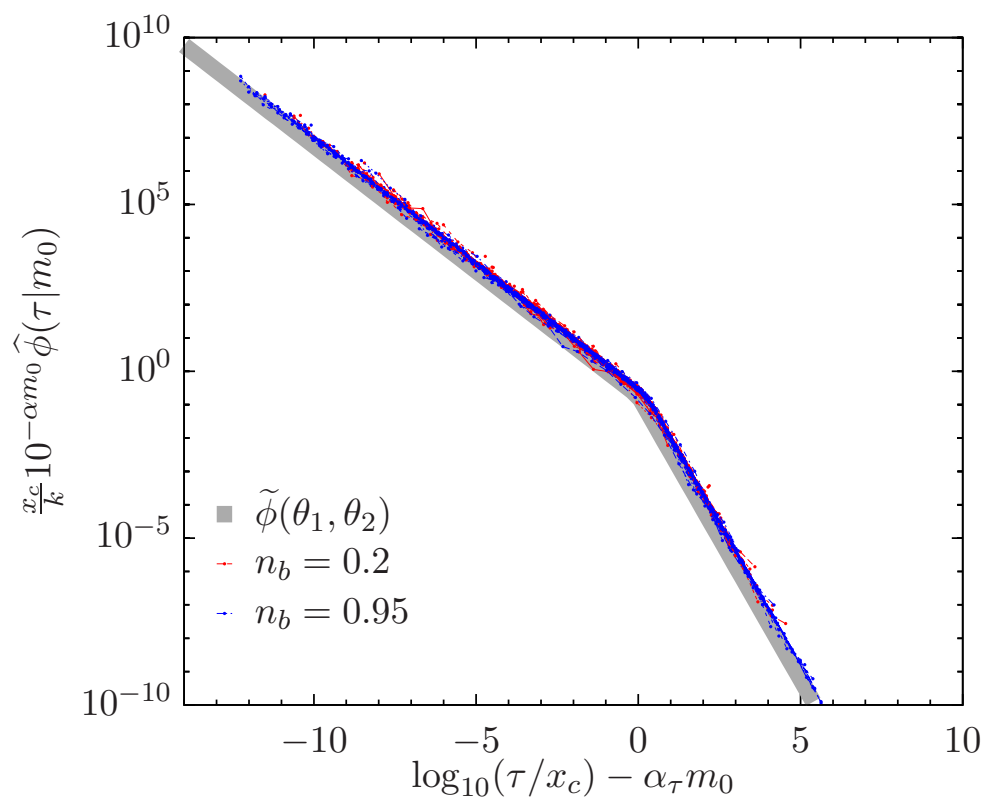

FiguRE 1: The triggering kernel recovered by the bare triggering rates measured in the simulations, exhibiting the scaling relation in $m_{0}, x_{c}$ and $n_{b}$ summarized by Eq. (16). Each curve (around 7 for each simulation) corresponds to the average 1st generation activity after mainshocks in different magnitude ranges $m<M_{i}<m+\Delta m$, with $\Delta m=0.5$. Six simulations are shown with values $x_{c}=1,0.0166$ and $10^{-8}$, respectively, and $n_{b}=0.2$ (in red) and 0.95 (in blue), respectively.

This is the form we focus on in the remainder of the paper, especially in the figures. Given the intensity of the process $\mu\left(t, \mathcal{H}_{t}\right)$ from Eq. (3), the terms $k, x, x_{c}$ and $\mu_{0}$ involve a temporal scale. Here, we select $\left\langle\mu_{0}(t)\right\rangle$ as our time unit. Thus, we express the parameter $x_{c}$ in units of the mean background rate: $\left[x_{c}\right]=\left\langle\mu_{0}(t)\right\rangle^{-1}$.

We implement the ETAS model with the modified temporal kernel as defined in Eq. (15) and performed simulations with the parameters estimated from the empirical data 48]: $\theta_{1}=0.25, \theta_{2}=0.7$ and a transition point $\tau_{c}=10^{\alpha_{\tau} m_{0}} x_{c}$. The magnitudes of the events are generated from Eq. (12) with $b=2.0$ and $m_{c}=3.252$. The productivity exponent of the kernel is set to $\alpha=0.55$ and $\alpha_{\tau}=0.5$. Thus the average number of events generated by a mainshock is $\left\langle N_{A S}\left(m_{0}\right)\right\rangle=k 10^{1.05 m_{0}}$. Combining Eqs. (16) and (14), the explicit dependence of the productivity term on the branching ratio $n_{b}$ reads:

$$
k\left(n_{b}\right)=\int d m_{0} \int d \tau \phi\left(\tau \mid m_{0}\right)=n_{b}\left(1-\frac{\alpha-\alpha_{\tau}}{b}\right) 10^{-\left(\alpha+\alpha_{\tau}\right) m_{c}}
$$

We generated sequences of $10^{5}$ background events for different values of $x_{c}$ and $n_{b}$. To highlight our main findings, we focus on two specific examples in the following: Low branching ratio with $n_{b}=0.2$ and high branching ration with $n_{b}=0.95$. The ratio between the transition point and average activity of unrelated events determines whether

\footnotetext{
${ }^{2}$ Please note that the magnitudes of $\mathrm{AE}$ events in the lab experiments are defined in a different way than for earthquakes and not directly comparable. Hence the difference in scales and $b$-values.
} 
the second power-law regime can be observed in the mean aftershock sequence rates or not. For example, the transition point $x_{c}$ is unobservable when the triggering rates $\phi\left(x_{c}\right)$ fall below the background rate $\mu_{0}$. Considering the parameters of our simulations, this happens for $x_{c}=\frac{\theta_{1} \theta_{2}}{\theta_{1}+\theta_{2}}=5 / 300 \sim 0.0167$ in our reduced units. We simulate the model with values of $x_{c}=1,5 / 300,10^{-8}$ such that the transition point is found above, around and below the background level. In order to evaluate the effect of time-independent vs time-dependent background rates, we impose $\mu_{0}(t) \sim t^{\sigma-1}$ by sampling the background events from a cumulative distribution: $\operatorname{CDF}\left(t_{i}\right)=t^{\sigma}$. Thus, a constant rate is sampled for $\sigma=1$ and quadratic increasing rate for $\sigma=3$, resembling the smooth increase of the rate observed at the beginning of AE experiments [4].

Fig. 1] shows the measured bare rates for all simulations to verify the scaling relation (16). Each line represents an average over all parent events with magnitude $m<$ $M_{i}<m+\Delta m$ in each simulation. Indeed, the numerical results reproduce the expected relation from Eq. (15), represented by the wide grey curve.

The compound triggering rates are also invariant with respect to $\tau_{c}=10^{-\alpha_{\tau} m_{0}} x_{c}$, which controls the temporal scale of the triggering with respect to the background rate, but it is sensitive to the productivity (given by $\int \phi\left(\tau \mid m_{0}\right) d m_{0}$ ), as stated in Eq. (6), and, thus, depends implicitly on $n_{b}$. The top panels of Fig. 2 show the average of the compound rates in mainshock magnitude windows of $\Delta m=0.5$, measured in the numerical simulations. The curves are scaled according to Eq. (16) in order to identify the deviations from the direct rates. For low branching ratio $\left(n_{b}=0.2\right)$ the compound rates are almost indistinguishable from the direct rates. This is not the case for higher branching ratios $\left(n_{b}=0.95\right)$ where we identify an exceedance of activity starting at $\tau \sim \tau_{c}$ and extending to higher values. Eq. (9) is only valid if the dependence on mainshock magnitude can be separated from the temporal kernel. In the modified ETAS model, the coupling imposed in the temporal scale $\tau_{c}\left(m_{0}\right)$ prevents this analytical approach. Yet, if we limit our analysis to the short-time power-law regime only (below $\tau_{c}$ ), we can at least provide an explanation for the exceedance point observed for $n_{b}=0.95 \mathrm{in} \mathrm{Fig.} \mathrm{2.} \mathrm{If} \mathrm{the} \mathrm{triggering} \mathrm{kernel} \mathrm{consisted}$ of a single power-law regime with exponent $\theta_{1}>0$ at all time-scales $\tau \rightarrow \infty$, the compound rates would always diverge and increase exponentially fast above a certain characteristic time $\tau^{*}$. When the branching ratio is high, the characteristic time $\tau^{*}$ is reached before the transition time $\tau_{c}$ towards the fast decaying regime. Since we now only consider the term: $\phi\left(\tau / \tau_{c}\right)=\left(\frac{\tau}{\tau_{c}}\right)^{\theta_{1}-1}$, we can separate the productivity from the pure temporal kernel and, following Eq. (9), obtain the resulting rate for $\tau \leq \tau_{c}$ :

$$
\Phi_{c}\left(\tau / \tau_{c}\right)=\frac{k}{x_{c}} 10^{\alpha m_{0}} \frac{\theta_{1} \theta_{2}}{\theta_{2}+\theta_{1}} \Gamma\left(\theta_{1}\right) \sum_{g=1}^{\infty} \frac{1}{\Gamma\left(g \theta_{1}\right)}\left(n_{b}\left(\frac{\tau}{\tau_{c}}\right)^{\theta_{1}}\right)^{g}
$$

with $\Gamma(\beta):=\int_{0}^{\infty} t^{\beta-1} \exp (-t) d t$. In Fig. 2 a,b the numerical solution from Eq. (18) (computed up to $g=50$ ) is plotted as a guide to the eye, revealing the trend to the deviation from Eq. (16) for high branching ratios. The solution for any value of $\theta_{2}>0$ and $n_{b}<1$ above the transition point $\tau=\tau_{c}$ should not differ significantly from the power-law decay discussed in Ref. 68.

\section{Inferring triggering rates in the modified ETAS model}

Without spatial information, the catalogs generated from a point process are given in a sequence of events $t_{i}, m_{i}$ and the direct and compound triggering rates cannot be measured 


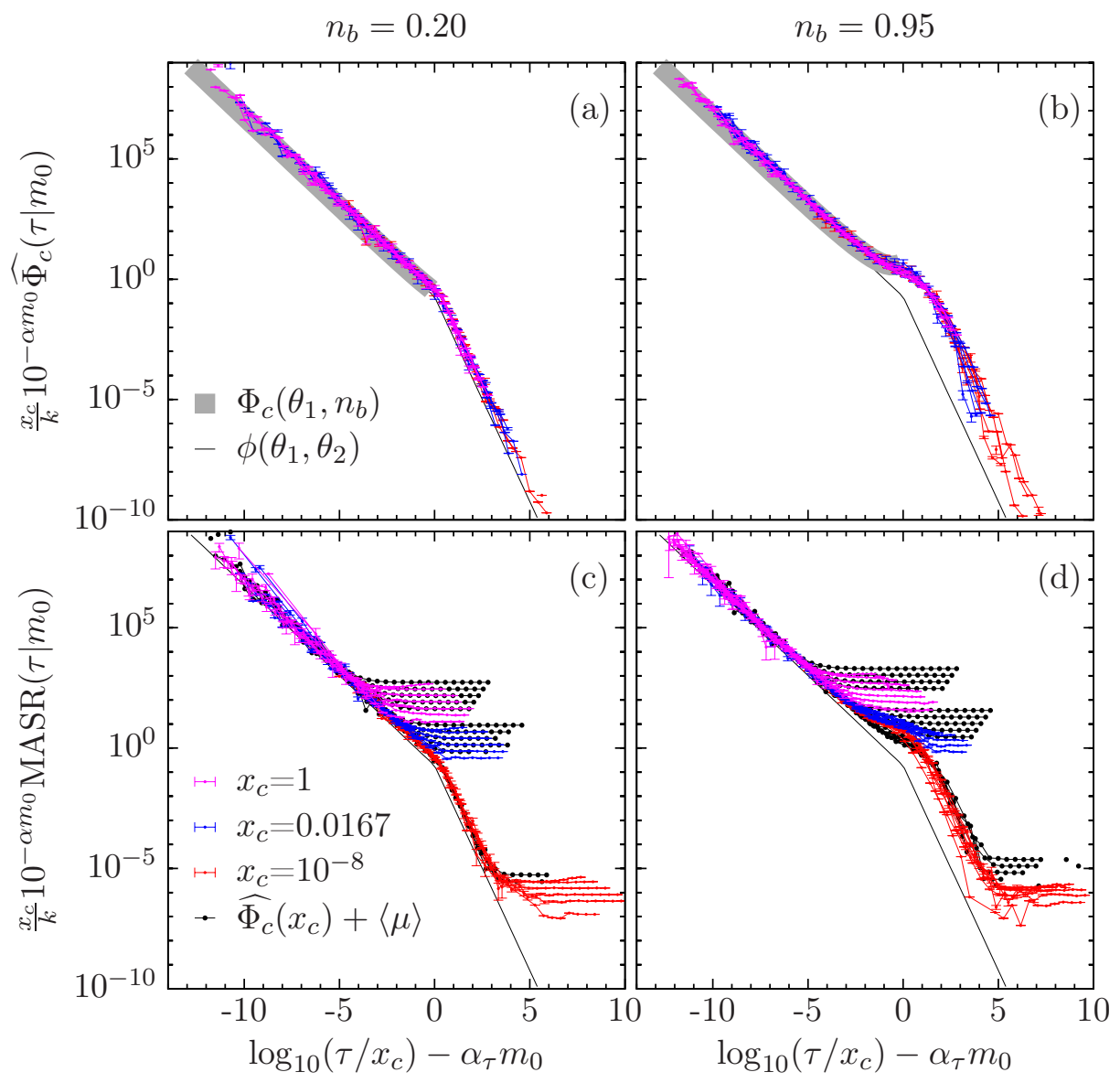

Figure 2: Rescaled compound triggering rates $(a, b)$ and mean aftershock sequence rates $(c, d)$ for the same simulations and mainshock magnitude bins as in Fig. 1. The scaling function for the triggering kernel is shown as thin black lines and the numerical solution of Eq. (18) is shown in grey. In $(c, d)$ the measured compound rates plus the average rate $\langle\mu\rangle$ from Eq. (11) are also plotted (black dots and lines). Error bars in $(c, d)$ correspond to one standard deviation for each individual sequence. The rate of events unrelated to the given triggering cascade $\langle\mu(t)\rangle$ (see Eq. (11)) decreases with the mainshock magnitude in the rescaled representation due to the term $10^{-\alpha m_{0}}$, giving rise to the variations in the plateaus observed at late times. 


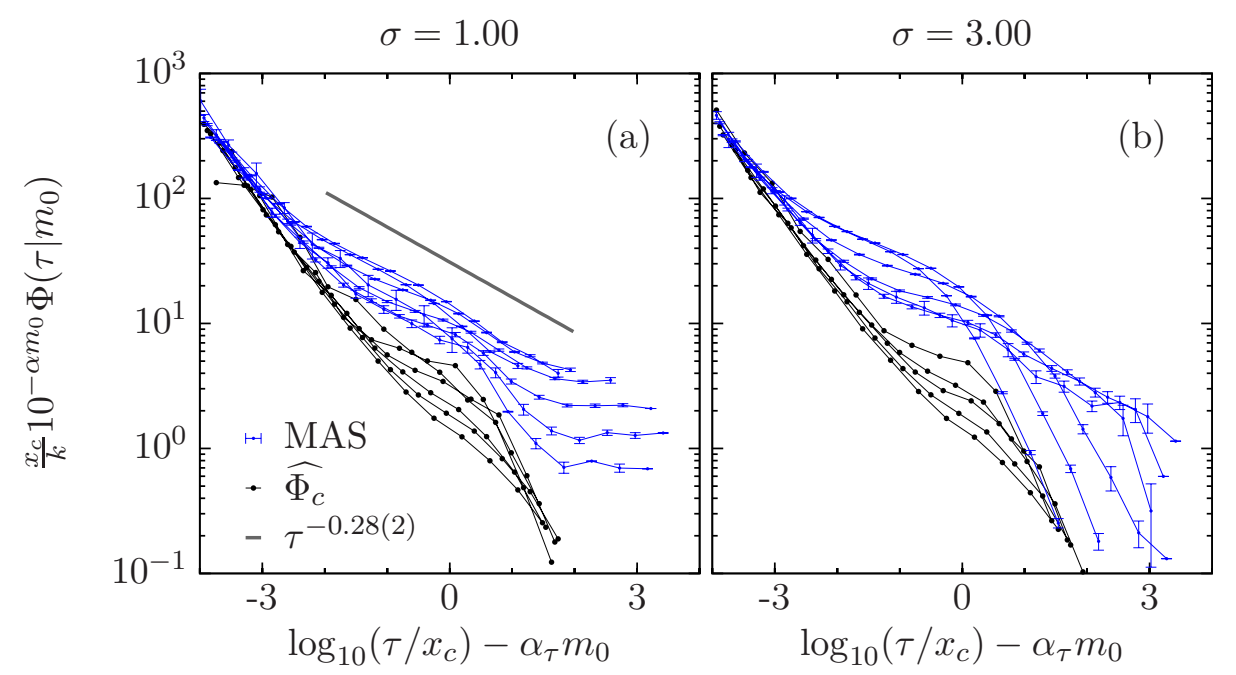

Figure 3: (a) Rescaled MASR (blue) and compound rates ( $\widehat{\Phi_{c}}$ in black) in a short interval around $\tau \sim \tau_{c}$ for $x_{c}=0.0167$ and $n_{b}=0.95$. The thick line represents a power-law with an average estimated value of $p=0.28(2)$ for the mean aftershock sequence rates. (b) Same as in (a) but for a time evolving background rate with a parabolic increase $(\sigma=3)$.

directly. Instead, we have to rely on the measurement of mean aftershock sequence rates (MASR) to infer the triggering kernel. In this section we present the results for synthetic catalogs generated from the modified ETAS model (Eq. (15)), and compare them with the actual direct and compound triggering rates.

Due to the increase in the aftershock rates with the magnitude of the mainshock as expressed by the productivity law, the overall intensity of the process as defined in Eq. (3) is typically dominated by an earlier large event or a more recent smaller one. To take advantage of this, we evaluate the mean aftershock sequence rates (MASR) as the activity after any event of any magnitude $M_{i}$ until the next event $j$ with magnitude $M_{j}>M_{i}$, in hopes to obtain long triggering sequences with reliable information. To obtain sufficient statistics, we average the MASR in mainshock magnitude windows $\left(m<M_{i}<m+\Delta m\right)$. We must normalize the sequences by the measurement range of each sequence: the time until an event is found with $M_{j}>M_{i}$, equivalent to the complementary cumulative distribution of waiting times $\operatorname{CCDF}\left(\delta, M_{k}>m\right)$. In Fig. 2]c,d the MASR measured in simulations are compared to the compound triggering rates $\left(\Phi_{c}(\tau, m)\right)$ with the addition of the time-averaged rate $\overline{\langle\mu(t)\rangle}$, presenting an approximation to the final expression of Eq. (11). Although the approximation is especially well-suited for low branching ratios, at $n_{b} \sim 1$, this assumption overestimates the contribution of unrelated events $\langle\mu(t)\rangle$ at late times. This is due to taking the time average since $\langle\mu(t)\rangle$ can significantly vary over time. Since triggered events are considered as mainshocks in the MASR, the rate of events triggered by independent branches within the same triggering tree are non-negligible. Although not directly triggered, this type of activity occurs predominantly at early times after the mainshock leading to a time-varying $\langle\mu(t)\rangle$. In general, we are able to recognize the double power-law kernel as long as the average rate $\langle\mu(t)\rangle$ is much lower than the triggering rate at the transition point $\frac{\theta_{1}+\theta_{2}}{\theta_{1} \theta_{2}} x_{c}$. Around and above this value, the average rate 
makes the secondary power-law regime unobservable and renders a single power-law decay in triggering rates resembling the standard Omori Utsu relation, but with an exponent value that is unphysically low, as was found in Refs. [7, 4, 10.

In the case of $\langle\mu(t)\rangle \sim \frac{\theta_{1}+\theta_{2}}{\theta_{1} \theta_{2}} x_{c}$ and $n_{b} \sim 1$, MASR (blue lines in Fig. 2]d) render an effective power-law behavior with a low exponent extending up to 5 decades around $x_{c}$. This behavior is a consequence of the interplay between the transition point, the rate of independent events, and the contribution of higher generation triggering. Fig. 3 shows this region in more detail for both a simulation with constant background rate (Fig. 3]a) and for $\sigma=3$ (Fig. 3. b). In the MASR for $\sigma=1$, we can fit the effective power-law with an exponent lower than 0.3 , not directly related to $\theta_{1}$ nor $\theta_{2}$, nor observed in the compound rates. The scaling relations of the triggering rates with $m_{0}$ are also affected. Thus, a blind estimation of Omori $(p)$ and the productivity $(\alpha)$ exponents limited within this interval by fitting and collapsing the MASR curves according to the scaling relations is unlikely to retrieve the right form of the triggering kernel.

Finally, we evaluate the distribution of waiting times (DWT) for different magnitude thresholds, as shown in Fig. 4. We compare the results obtained with (a) the uniform $(\sigma=1)$ and (b) time-dependent $(\sigma=3)$ background rate, for different values of $x_{c}$ and $n_{b}=0.95$. No significant differences are found for $n_{b}=0.20$ (not shown). The distributions are scaled with the mean waiting time for each threshold, following the scaling relation expected for a Poisson process and observed also for other processes such as seismicity [84, 46] and rock fracture 45, for example. In the standard ETAS model, the situation is more complicated [85, 86]. This is also true for the modified ETAS model we consider here. Due to the additional scaling parameter $\left(\alpha_{\tau}\right)$ the collapsing of the curves to a single scaling function can only be fulfilled over certain ranges. If the events were independent, from the background sampling one would expect an exponential distribution in the DWT for $\sigma=1$ and a power law decay with exponent: $\frac{2 \sigma-1}{\sigma-1}=2.5$ for $\sigma=3$. Instead, both behaviors are only found for waiting times longer than the typical waiting time of background events $(\delta \sim\langle\delta\rangle)$. Below these times, the distribution is dominated by the triggering process, returning the power-law exponents predicted from the relation $2-1 / p$ : 0.67 for the regime with $p=0.75$ and 1.41 for the regime $p=1.7$, see Fig. 4 .

\section{Discussion}

As expected, the branching ratio, the ratio between the power-law transition and the background rate are essential to understand the results of MASR in terms of the triggering kernel. The existence of a characteristic scale in the temporal triggering kernel offers a plausible explanation to the detection of effective Omori exponents lower than one in unlocalized catalogs of acoustic emission during mechanical processes [7, 44, 73, 10, 78, 87] and calorimetry in structural phase transitions [4. In the specific case of the failure of porous materials under compression [7, 44, an effective Omori exponent $p \sim 0.7$ was observed using MASR, compatible with the short time power-law regime found in localized catalogs 48, and the MASR of the modified ETAS model (Fig. 2) for transition values $x_{c} \gtrsim \frac{\theta_{1} \theta_{2}}{\theta_{1}+\theta_{2}}$. The explanation derived from the modified ETAS models is that the second power law regime is hidden by the background rate. As a consequence, the estimation of the productivity term $k\left(m_{0}\right)$ will neglect the existance of a scaling relation in the temporal axis. Thus, only a single exponent can be estimated by collapsing the curves $\phi\left(\tau \mid m_{0}\right)$. In the modified ETAS model, if the second power-law is not observed in the MASR, one would find a scaling relation $k\left(m_{0}\right) \sim 10^{\alpha^{\prime} m_{0}}$, but this measured exponent $\alpha^{\prime}$ does not correspond to the scaling parameter $\alpha$, nor the productivity exponent $\widetilde{\alpha}=\alpha+\alpha_{\tau}$. Instead it corresponds to an intermediate value $\alpha^{\prime}=\alpha+\alpha_{\tau}\left(1-\theta_{1}\right)=\widetilde{\alpha}-\theta_{1} \alpha_{\tau}$. This 


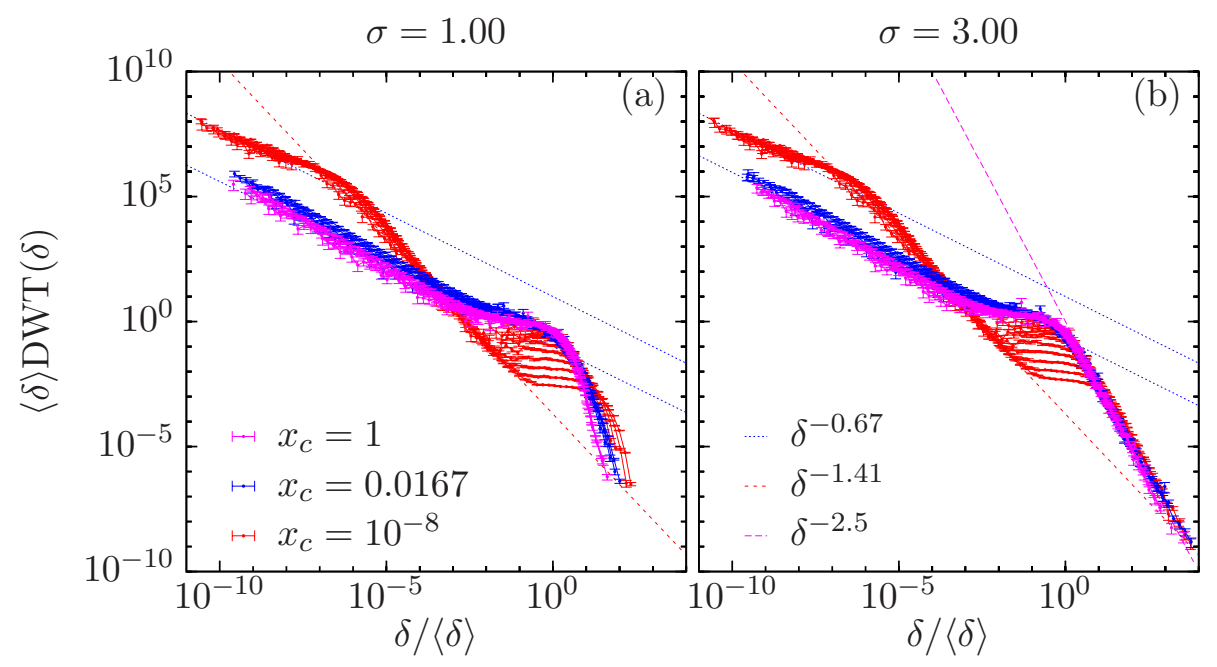

FiguRE 4: Distribution of waiting times rescaled by the average value for $n_{b}=0.95$ and $x_{c}$ above, below and at the background level, and for (a) a uniform background rate $(\sigma=1)$ and $(b)$ for a background rate parabolically rising from $\mu_{0}=0(\sigma=3)$. Each line represents a different magnitude threshold $m_{c}=3.25,3.50, \ldots, 6.5$. The expected power-law behaviors are represented as a guide to the eye. One sigma error bars are shown.

relation directly follows from Eqs. (15) and (16) if one only considers the regime $x \leq x_{c}$. The specific value of $\alpha^{\prime}=0.925$ for our simulations (and consistent with the experiments in [48) is, however, different from the value $\alpha^{\prime} \approx 0.5$ observed during the failure of porous materials under compression [7] using MASR. Provided the validity of the modified ETAS model, this suggests that the exponents $\alpha$ and $\alpha_{\tau}$ are not universal across rock fracture experiments. Assuming positive values of $\alpha, \alpha_{\tau}$ and $\theta_{1}=0.25$, both exponents are however limited within the range $0 \leq \alpha \leq 2$ and $0 \leq \alpha_{\tau} \leq 2 / 3$.

The distribution of waiting times in experimental data usually exhibits a sharp transition between two power-law regimes [76, 7, 78. The power-law regime observed for long waiting times is consistent with the temporal variations of the background rate. Our modified ETAS model can reproduce both observations if the maximum background rate is comparable to the rate at the transition point between the two power laws in the triggering kernel, i.e. $x_{c}=0.0167$ in our simulations. The long time regime of the triggering kernel is only observable before the regime dominated by the background rate for $x_{c}<0.0167$, as shown in Fig. 4 The absence of the secondary triggering regime for $x_{c} \gtrsim 0.0167$ is also consistent with the above mentioned absence of the secondary regime above the background rate in the experimentally measured MASR.

Finally, the exact mathematical relation between the $p$-value of the direct triggering rates and the power-law exponent in the waiting time distribution $(2-1 / p)$ is not consistent with the exponents measured using MASR in some experiments [7, 78. While one might expect that this is related to the presence of a high branching ratio [68, this is not supported by our findings for the modified ETAS model considered here and remains an open question. 


\section{Conclusions}

The measurement of triggering rates is a non-trivial problem, even in simplified branching processes. Due to limitations in the acquisition systems, we often must rely on the indirect measurement of mean aftershock sequence rates (MASR) to infer the original triggering kernel. The performance of this technique will depend specifically on the background rate and the branching ratio. We can retrieve the triggering kernel with a good precision whenever the individual triggering trees can be separated at low background rates and if secondary triggering can be neglected due to low branching ratio. But, in general, one should expect a strong superposition of independent and secondary activity.

When the data is sampled from a triggering kernel with characteristic time scales such as proposed in 48, for rock fracture and implemented in this work in the form of a modified ETAS model - the interplay between the characteristic scale of the triggering and the background rate can render non-scaling regimes in both the measurement of triggering rates using MASR and the distribution of waiting times. As a specific case, if the characteristic time scale and the time scale of the background activity are comparable we find a crossover regime similar to another power law. Yet, its exponent cannot be trivially associated with the underlying parameters of the modified ETAS model.

In more general terms, our study here shares light on the problem of separating overlapping triggering cascades or branching trees from limited information to establish the underlying causal relationships, which is not specific to slip and fracture events. Indeed, another prime example is neuronal activity for which such investigations are still at the very beginning [88, 89].

\section{References}

[1] J. P. Sethna, K. A. Dahmen, and C. R. Myers. Crackling noise. Nature (London), 410:242, 2001.

[2] G. Durin and S. Zapperi. In G. Bertotti and I. Mayergoyz, editors, The Science of Hysteresis, volume II., page 181. Academic Press, San Diego, 2006.

[3] S. Papanikolaou, F. Bohn, R. L. Sommer, G. Durin, S. Zapperi, and J. P. Sethna. Universality beyond power laws and the average avalanche shape. Nature Physics, 7:316, 2011.

[4] Jordi Baró, José-María Martín-Olalla, Francisco Javier Romero, María Carmen Gallardo, Ekhard K H Salje, Eduard Vives, and Antoni Planes. Avalanche correlations in the martensitic transition of a $\mathrm{Cu}-\mathrm{Zn}-\mathrm{Al}$ shape memory alloy: analysis of acoustic emission and calorimetry. Journal of Physics: Condensed Matter, 26(12):125401, 2014.

[5] D. Bonamy and E. Bouchaud. Failure of heterogeneous materials: A dynamic phase transition? Physics Reports, 498:1, 2011.

[6] A. Tantot, S. Santucci, O. Ramos, S. Deschanel, M.-A. Verdier, E. Mony, Y. Wei, S. Ciliberto, L. Vanel, and P. C. F. Di Stefano. Sound and light from fractures in scintillators. Physical Review Letters, 111:154301, 2013.

[7] Jordi Baró, Steve Dixon, Rachel S Edwards, Yichao Fan, Dean S Keeble, Lluís Mañosa, Antoni Planes, and Eduard Vives. Simultaneous detection of acoustic emission and Barkhausen noise during the martensitic transition of a Ni-Mn-Ga magnetic shape-memory alloy. Physical Review B, 88(17):174108, 2013.

[8] F. Kun, I. Varga, S. Lennartz-Sassinek, and I. G. Main. Rupture cascades in a discrete element model of a porous sedimentary rock. Physical Review Letters, 112:065501, 2014. 
[9] T. Mäkinen, A. Miksic, M. Ovaska, and Mikko J. Alava. Avalanches in wood compression. Physical Review Letters, 115:055501, 2015.

[10] H. V. Ribeiro, L. S. Costa, L. G. A. Alves, P. A. Santoro, S. Picoli, E. K. Lenzi, and R. S. Mendees. Analogies between the cracking noise of ethanol-dampened charcoal and earthquakes. Physical Review Letters, 115:025503, 2015.

[11] Y. Ben-Zion. Collective behavior of earthquakes and faults: Continuum-discrete transitions, progressive evolutionary changes, and different dynamic regimes. Review of Geophysics, 46:RG4006, 2008.

[12] C. Gu, G. St-Yves, and J. Davidsen. Spiral wave chimeras in complex-oscillatory and chaotic systems. Physical Review Letters, 111:134101, 2013.

[13] Jörn Davidsen and Marco Baiesi. Self-similar aftershock rates. Physical Review E, 94(2):022314, 2016.

[14] Samira Maghsoudi, David W Eaton, and Jörn Davidsen. Nontrivial clustering of microseismicity induced by hydraulic fracturing. Geophysical Research Letters, 43(20), 2016.

[15] M. Baiesi, M. Paczuski, and A. L. Stella. Intensity thresholds and the statistics of the temporal occurrence of solar flares. Physical Review Letters, 96:051103, 2006.

[16] L. de Arcangelis, C. Godano, E. Lippiello, and M. Nicodemi. Universality in solar flare and earthquake occurrence. Physical Review Letters, 96:051102, 2006.

[17] N. R. Moloney and J. Davidsen. Extreme bursts in the solar wind. Geophysical Research Letters, 38:L14111, 2011.

[18] N. R. Moloney and J. Davidsen. Stationarity of extreme bursts in the solar wind. Physical Review E, page submitted, 2013.

[19] D. L. Turcotte. Self-organized criticality. Reports on Progress in Physics, 62:1377, 1999.

[20] P. Bak. How nature works. Copernicus, New York, 1996.

[21] J. P. Crutchfield and P. Schuster. Evolutionary Dynamics: Exploring the Interplay of Selection, Accident, Neutrality, and Function. Oxford University Press US, NY, NY, 2003.

[22] B. Drossel. Biological evolution and statistical physics. Advances in Physics, 50:209, 2001.

[23] N. Friedman, S. Ito, B. A. W. Brinkman, M. Shimono, R. E. L. DeVille, K. A. Dahmen, J. M. Beggs, and T. C. Butler. Universal critical dynamics in high resolution neuronal avalanche data. Physical Review Letters, 108:208102, 2012.

[24] Mohammad Yaghoubi, Ty de Graaf, Javier G. Orlandi, Fernando Girotto, Michael Colicos, and Jörn Davidsen. Non-trivial neuronal avalanche dynamics in developing neuronal cultures. (preprint), 2017.

[25] Didier Sornette, Anders Johansen, and Jean-Philippe Bouchaud. Stock market crashes, precursors and replicas. Journal de Physique I, 6(1):167-175, 1996.

[26] J. D. Farmer, D. E. Smith, and M. Shubik. Is economics the next the next physical sciences? Physics Today, 58(9):37, 2005.

[27] F. Lillo and R. N. Mantegna. Power-law relaxation in a complex system: Omori law after a financial market crash. Physical Review E, 68:016119, 2003.

[28] P. Weber, F. Wang, I. Vodenska-Chitkushev, S. Havlin, and H. E. Stanley. Relation between volatility correlations in financial markets and Omori processes occurring on all scales. Physical Review E, 76:016109, 2007. 
[29] A. M. Petersen, F. Wang, S. Havlin, and H. E. Stanley. Market dynamics immediately before and after financial shocks: Quantifying the Omori, productivity, and Bath laws. Physical Review E, 82:036114, 2010.

[30] F. M. Siokis. Stock market dynamics: Before and after stock market crashes. Physica A, 391:1315, 2012.

[31] P. Klimek, W. Bayer, and S. Thurner. The blogosphere as an excitable social medium: Richter's and omori's law in media coverage. Physica A, 390:3870, 2011.

[32] J. Weiss and M. Carmen Miguel. Dislocation avalanche correlations. Materials Science and Engineering A, 387-389:292, 2004.

[33] R. Crane and D. Sornette. Robust dynamic classes revealed by measuring the response function of a social system. Proceedings National Academy of Sciences U.S.A., 105:15649, 2008.

[34] D. Sornette and S. Utkin. Limits of declustering methods for disentangling exogenous from endogenous events in time series with foreshocks, main shocks, and aftershocks. Physical Review E, 79:061110, 2009.

[35] C. Gu, A. Y. Schumann, M. Baiesi, and J. Davidsen. Triggering cascades and statistical properties of aftershocks. Journal of Geophysical Research, 118:4278, 2013.

[36] S. Hainzl, J. Moradpour, and J. Davidsen. Static stress triggering explains the empirical aftershock distance decay. Geophysical Research Letters, 41:8818, 2014.

[37] M. Stojanova, S. Santucci, L. Vanel, and O. Ramos. High frequency monitoring reveals aftershocks in subcritical crack growth. Physical Review Letters, 112:115502, 2014.

[38] T. Utsu, Y. Ogata, and R. S. Matsu'ura. The centenary of the Omori formula for a decay law of aftershock activity. J. Phys. Earth, 43:1, 1995.

[39] J. Moradpour, S. Hainzl, and J. Davidsen. Nontrivial decay of aftershock density with distance in Southern California. Journal of Geophysical Research, 119:5518, 2014.

[40] J. Davidsen, C. Gu, and M. Baiesi. Generalized Omori-Utsu law for aftershock sequences in southern California. Geophysical Journal International, 201:965, 2015.

[41] T. H. W. Goebel, C. G. Sammis, T. W. Becker, G. Dresen, and D. Schorlemmer. A comparision of seismicity characteristics and fault structure in stick-slip experiments and nature. Pure and Applied Geophysics, 172:2247, 2013.

[42] F. Omori. On the aftershocks of earthquakes. Journal of College Science, Imperial University of Tokyo, 7:111, 1894.

[43] Pedro O Castillo-Villa, Jordi Baró, Antoni Planes, Ekhard K H Salje, Pathikumar Sellappan, Waltraud M Kriven, and Eduard Vives. Crackling noise during failure of alumina under compression: the effect of porosity. Journal of Physics: Condensed Matter, 25(29):292202, 2013.

[44] Guillaume F. Nataf, Pedro O. Castillo-Villa, Jordi Baró, Xavier Illa, Eduard Vives, Antoni Planes, and Ekhard K. H. Salje. Avalanches in compressed porous Si02-based materials. Physical Review E, 90(2):022405, 2014.

[45] J. Davidsen, S. Stanchits, and G. Dresen. Scaling and universality in rock fracture. Physical Review Letters, 98:125502, 2007.

[46] Jörn Davidsen and Grzegorz Kwiatek. Earthquake Interevent Time Distribution for Induced Micro-, Nano-, and Picoseismicity. Physical Review Letters, 110(6):68501, feb 2013.

[47] SD Goodfellow and RP Young. A laboratory acoustic emission experiment under in situ conditions. Geophysical Research Letters, 41(10):3422-3430, 2014.

[48] Elli-Maria Charalampidou Thomas Goebel Sergei Stanchits Marc Rück Jörn Davidsen, Grzegorz Kwiatek and Georg Dresen. Triggering proccesses in rock fracture. 
[49] J. Davidsen, P. Grassberger, and M. Paczuski. Networks of recurrent events, a theory of records, and application to finding causal signatures in seismicity. Physical Review E, 77:066104, 2008.

[50] D. Marsan and O. Lengliné. Extending earthquake' reach through cascading. Science, 319:1076, 2008.

[51] I. Zaliapin and Y. Ben-Zion. Earthquake clusters in southern California, I: Identification and stability. Journal of Geophysical Research, 118:2847, 2013.

[52] Agnès Helmstetter. Is Earthquake Triggering Driven by Small Earthquakes? Physical Review Letters, 91(5):58501, 2003.

[53] Y. Y. Kagan and L. Knopoff. Statistical short-term earthquake prediction. Science, 236:1563, 1987.

[54] Yosihiko Ogata. Seismicity analysis through point-process modeling: A review. In Seismicity patterns, their statistical significance and physical meaning, pages 471-507. Springer, 1999.

[55] Daryl J Daley and David Vere-Jones. An introduction to the theory of point processes: volume II: general theory and structure, volume 2. Springer Science \& Business Media, 2007.

[56] Álvaro Corral. Point-occurrence self-similarity in crackling-noise systems and in other complex systems. Journal of Statistical Mechanics: Theory and Experiment, 2009(01):P01022, 2009.

[57] Alan G Hawkes and David Oakes. A cluster process representation of a self-exciting process. Journal of Applied Probability, pages 493-503, 1974.

[58] Agnes Helmstetter, Yan Y Kagan, and David D Jackson. Comparison of short-term and time-independent earthquake forecast models for southern california. Bulletin of the Seismological Society of America, 96(1):90-106, 2006.

[59] Vladimir Filimonov and Didier Sornette. Quantifying reflexivity in financial markets: Toward a prediction of flash crashes. Physical Review E, 85(5):056108, 2012.

[60] K. F. Tiampo and R. Shcherbakov. Seismicity-based earthquake forecasting techniques: Ten years of progress. Tectonophysics, 522:89, 2012.

[61] Emmanuel Bacry, Iacopo Mastromatteo, and Jean-François Muzy. Hawkes processes in finance. Market Microstructure and Liquidity, 1(01):1550005, 2015.

[62] Paolo Sibani and H Jeldtoft Jensen. Intermittency, aging and extremal fluctuations. EPL (Europhysics Letters), 69(4):563, 2005.

[63] Nicholas J van der Elst, Emily E Brodsky, and Thorne Lay. Remote triggering not evident near epicenters of impending great earthquakes. Bulletin of the Seismological Society of America, 103(2B):1522-1540, 2013.

[64] E. A. Jagla. Aftershock production rate of driven viscoelastic interfaces. Physical Review E - Statistical, Nonlinear, and Soft Matter Physics, 90(4):1-8, 2014.

[65] Sanja Janićević, Lasse Laurson, Knut Jørgen Måløy, Stéphane Santucci, and Mikko J Alava. Interevent correlations from avalanches hiding below the detection threshold. Physical Review Letters, 117(23):230601, 2016.

[66] I. Zaliapin, A. Gabrielov, V. Keilis-Borok, and H. Wong. Clustering analysis of seismicity and aftershock identification. Physical Review Letters, 101:018501, 2008.

[67] S Hainzl, A Christophersen, D Rhoades, and D Harte. Statistical estimation of the duration of aftershock sequences. Geophysical Journal International, 205(2):1180$1189,2016$. 
[68] Agnès Helmstetter and Didier Sornette. Subcritical and supercritical regimes in epidemic models of earthquake aftershocks. Journal of Geophysical Research: Solid Earth, 107(B10):ESE 10-1-ESE 10-21, 2002.

[69] Agnès Helmstetter, Didier Sornette, and Jean-Robert Grasso. Mainshocks are aftershocks of conditional foreshocks: How do foreshock statistical properties emerge from aftershock laws. Journal of Geophysical Research: Solid Earth (1978-2012), 108(B1), 2003.

[70] Nicholas J Van Der Elst and Emily E Brodsky. Connecting near-field and far-field earthquake triggering to dynamic strain. Journal of Geophysical Research: Solid Earth, 115(B7), 2010.

[71] Hongguang Bi, Gerhard Börner, and Yaoquan Chu. Correlations in the absorption lines of the quasar Q0420-388. Astronomy and Astrophysics, 218:19-23, 1989.

[72] V Carbone, L Sorriso-Valvo, A Vecchio, F Lepreti, P Veltri, P Harabaglia, and I Guerra. Clustering of polarity reversals of the geomagnetic field. Physical Review Letters, 96(12):128501, 2006.

[73] Jordi Baró, Antoni Planes, Ekhard K H Salje, and Eduard Vives. Fracking and labquakes. Philosophical Magazine, 6435(September):1-11, 2016.

[74] Jordi Baró, Peter Shyu, Siyuan Pang, Iwona M Jasiuk, Eduard Vives, Ekhard KH Salje, and Antoni Planes. Avalanche criticality during compression of porcine cortical bone of different ages. Physical Review E, 93(5):053001, 2016.

[75] M S Wheatland. The Origin of the Solar Flare Waiting-Time Distribution. The AstroPhysical Journal Letters, 536(2):L109, 2000.

[76] Alvaro Corral. Local distributions and rate fluctuations in a unified scaling law for earthquakes. Physical Review E, 68(3):035102, 2003.

[77] Robert Shcherbakov, Gleb Yakovlev, Donald L Turcotte, and John B Rundle. Model for the distribution of aftershock interoccurrence times. Physical Review Letters, 95(21):218501, 2005.

[78] T. Mäkinen, a. Miksic, M. Ovaska, and Mikko J. Alava. Avalanches in Wood Compression. Physical Review Letters, 115(5):055501, 2015.

[79] Beno Gutenberg and Charles F Richter. Frequency of earthquakes in California. Bulletin of the Seismological Society of America, 34(4):185-188, 1944.

[80] G. Kwiatek, K. Plenkers, M. Nakatani, Y. Yabe, G. Dresen, and JAGUARS-Group. Frequency-magnitude characteristics down to magnitude -4.4 for induced seismicity recorded at Mponeng gold mine, South Africa. Bulletin of the Seismological Society of America, 100:1165, 2010.

[81] J. Davidsen and A. Green. Are earthquake magnitudes clustered? Physical Review Letters, 106:108502, 2011.

[82] J. Davidsen, G. Kwiatek, and G. Dresen. No evidence of magnitude clustering in an aftershock sequence of nano- and picoseismicity. Physical Review Letters, 108:038501, 2012.

[83] Yosihiko Ogata. Statistical models for earthquake occurrences and residual analysis for point processes. Journal of the American Statistical Association, 83(401):9-27, 1988.

[84] Á. Corral. Long-term clustering, scaling, and universality in the temporal occurrence of earthquakes. Physical Review Letters, 92:108501, 2004.

[85] S. Touati, M. Naylor, I. G. Main, and M. Christie. Masking of earthquake triggering behavior by a high background rate and implications for epidemic-type aftershock sequence inversions. Journal of Geophysical Research, 116:B03304, 2011. 
[86] Eugenio Lippiello, Álvaro Corral, Milena Bottiglieri, Cataldo Godano, and Lucilla de Arcangelis. Scaling behavior of the earthquake intertime distribution: Influence of large shocks and time scales in the omori law. Physical Review E, 86(6):066119, 2012.

[87] Leandro S Costa, Ervin K Lenzi, Renio S Mendes, and Haroldo V Ribeiro. Extensive characterization of seismic laws in acoustic emissions of crumpled plastic sheets. EPL (Europhysics Letters), 114(5):59002, 2016.

[88] Rashid V Williams-Garcia, John M Beggs, and Gerardo Ortiz. Unveiling causal activity of complex networks. arXiv preprint arXiv:1603.05659, 2016.

[89] Javier G. Orlandi, Jordi Soriano, Enrique Alvarez-Lacalle, Sara Teller, and Jaume Casademunt. Noise focusing and the emergence of coherent activity in neuronal cultures. Nature Physics, 9(9):582-590, 2013. 